\title{
Reduced Formulae for Vibration of Continuous Beams with Application on Moving Loads
}

\author{
T.G. Konstantakopoulos, I.G. Raftoyiannis and G.T. Michaltsos*
}

Department of Civil Engineering, National Technical University of Athens, Greece

\begin{abstract}
The present paper concludes via an analytical method to reduced formulae for determining the eigenfrequencies and eigenmodes of multi-span continuous beams with spans of different lengths and bending rigidities in a very easy and efficient way. The rigorous determination of eigenfrequencies and eigenmodes allows us to focus on the derivation of the time function for the forced vibrating beam subjected to the action of moving loads. The dynamic response of such beams subjected to a load moving with constant velocity is thoroughly studied. The analysis is carried out by the modal superposition method. Numerical examples are presented to verify the applicability of the presented formulae.
\end{abstract}

Keywords: Continuous beams, moving loads, free vibration, reduced formulae.

\section{INTRODUCTION}

The dynamic behavior of beams such as railway bridges subjected to moving loads or moving masses has been investigated in detail over the past century. An extensive bibliography on this area is available in the well-known work of Frýba [1].

Despite the above-mentioned extensive bibliography on the vibrations of single-span beams, the dynamic behavior of continuous (multi-span) beams has not been thoroughly studied since the solution of the problem becomes evidently more cumbersome as the number of beam spans increases.

There are two commonly used approaches for computing the eigenfrequencies of continuous beams, i.e. the analytical method and the finite element method.

In the finite element method, a large number of dynamic elements is required in order to sufficiently model a continuous beam and generate the full frequency spectrum by solving the obtained linear eigenvalue matrix equation. The precision depends on the number of finite elements used in the model and a parametric analysis requires the constructions of several finite element models.

In the analytical method, each span of the continuous beam is considered as an individual one and both the solution of a fourth-order differential equation and its corresponding modal shape is a priori known. By using the compatibility and equilibrium conditions at each junction between adjacent spans, a homogeneous linear system of equations is obtained, which provides the eigenvalues problem of the beam and thus, the spectrum of eigenfrequencies.

Special attention should be paid to Inglis [2] study, who employed harmonic analysis for the solution of all

*Address correspondence to this author at the Department of Civil Engineering National Technical University of Athens, 9 Iroon Polytechneiou Str, Zografou Campus, Athens, 15780 Greece; Tel: +30-210-7722482;

Fax:+30-210-7723443; E-mail: michalts@central.ntua.gr practically important cases of railway bridge subjected to loads resulting from the passage of steam moving trains. Hillerborg [3], by employing the Fourier method and other differential methods, provided a first solution to the problem of one load moving with constant speed on a system supported by elastic springs. Further progress to this direction became possible with the use of computers. Biggs et al. [4] solved the problem using the method by Inglis and the obtained solutions were applied to bridge dynamics.

Regarding relative experimental research, AASHO $[5,6]$ Road Test was an extensive experimental program conducted by the Highway Research Board (HRB). The main goal of the experiments was the investigation of behavior of paving and bridges under the action of a large number of dynamic loads, the conduct of measurements and the drawn of conclusions. These experiments on dynamic investigation of bridges were carried out at the University of Illinois. Oehler [7] published the results of experiments in 15 road bridges, where always the same experimental vehicle, a 3-axes track, was used.

Today, with the advanced development of technology, one can deal more easily with such problems, as Bily [8] in Prague and Hymay in Ottawa, who employed threedimensional models. Research on moving loads is still carried out in the Universities of Illinois (Urbana), Michigan State (East Lansing), Northwestern (Evanston), Standard, M.I.T. and elsewhere. In Europe, similar research is carried out in Poland and Switzerland, mainly on railway bridges, in France on road bridges, while in Germany they are dealing with both types.

The corresponding bibliography on continuous beams is rather poor though. There are a lot of investigations for the study of the dynamic behavior of continuous beams with two or three spans. The following studies on the dynamics of continuous beams are worth mentioning. Dmitriev [9] presented a method for determining the dynamic deflections of multi-span beams but with equal spans. Kong \& Cheung [10] studied the free vibration of a continuous beam using the 
Ritz method within the context of the Euler beam theory. Luo [11] studied the lateral vibration of an axially loaded infinite continuous beam. Munirudrappa \& Dhruvaraja Iyengar [12] presented a computer program for the solution of the equations governing the motion of a continuous-span highway bridge. Ichikawa et al. [13] presented a method for the direct determination of the natural modes by using the method of eigenfunctions expansion. Greco \& Santini [14] studied the dynamic analysis of a continuous beam using an extension of the complex mode superposition method. Samaan et al. [15] studied curved continuous multiple-box girder bridges. Mehri et al. [16] studied the problem of a continuous beam and presented a solution employing the Green's functions.

The present work concludes through an analytical method to reduced formulae for determining the eigenfrequencies and eigenfunctions of multi-span continuous beams with spans of different lengths and rigidities. The presented approach is called "Reduced Formulae" since the expressions for a $v$-span continuous beam are reduced to the expressions corresponding to a $(v-1)$-span continuous beam and so on.

The dynamic response of a continuous beam subjected to a load moving with constant velocity is thoroughly studied. A model of moving load (although non-realistic for some cases met in common praxis) is employed to show the rigorous handing of the proposed formulae. The analysis is carried out by the modal superposition method. Numerical examples are presented to illustrate the applicability of the presented formulae.

\section{MATHEMATICAL FORMULATION}

Let us consider the multi-span beam of Fig. (1) made from a homogeneous, isotropic and linearly elastic material with modulus of elasticity $\mathrm{E}$. The continuous beam consists of $v$ spans with lengths $\ell_{i}$, cross-section areas $A_{i}$, moments of inertia $I_{i}$ and masses per unit length $m_{i}$, with $i=1,2, \ldots, v$ and is resting on pinned supports.

\section{The Free Vibrating Beam}

Neglecting the influence of longitudinal motion, the equations of motion of the freely vibrating beam are:

$$
\mathrm{EI}_{\mathrm{i}} \mathrm{w}_{\mathrm{i}}^{\prime \prime \prime}\left(\mathrm{x}_{\mathrm{i}}, \mathrm{t}\right)+\eta_{\mathrm{i}} \dot{\mathrm{w}}_{\mathrm{i}}\left(\mathrm{x}_{\mathrm{i}}, \mathrm{t}\right)+\mathrm{m}_{\mathrm{i}} \ddot{\mathrm{w}}_{\mathrm{i}}\left(\mathrm{x}_{\mathrm{i}}, \mathrm{t}\right)=0
$$

where $\mathrm{i}=1,2, \ldots, v$, and the prime indicates differentiation with respect to $\mathrm{x}$, the dot indicates differentiation with respect to time $t$, and $\eta$ is the damping coefficient.

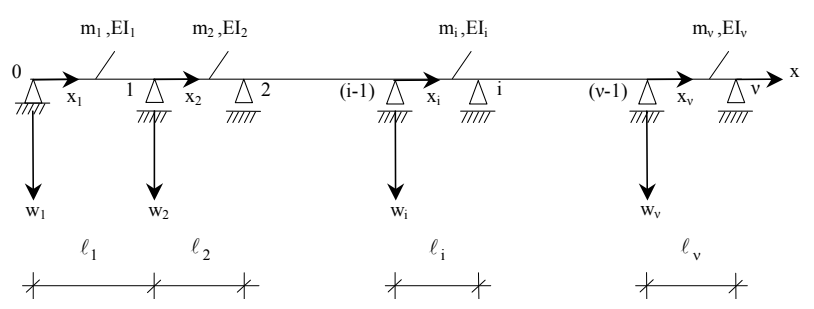

Fig. (1). Geometry and sign convention of a $v$-span continuous beam.
The factor $\eta_{i}$ is the damping coefficient of the variant Kelvin-Voigt model. Theoretically, it is $\eta_{i}=\eta_{i}(x)$, but practically one can consider that $\eta_{i}=$ const. along $x$, depending only on the material of the beam.

We are searching for a solution in the form of separate variables:

$\mathrm{w}_{\mathrm{i}}\left(\mathrm{x}_{\mathrm{i}}, \mathrm{t}\right)=\mathrm{X}_{\mathrm{i}}\left(\mathrm{x}_{\mathrm{i}}\right) \cdot \mathrm{T}(\mathrm{t})$

Introducing eq(2) into eq(1), we obtain

$\frac{E I_{i} X_{i}{ }^{\prime \prime \prime \prime}}{m_{i} X_{i}}=-\frac{\ddot{T}+\frac{\eta_{i}}{m_{i}} \dot{T}}{T}=\omega^{2}$

The above leads to the following two equations:

$$
\begin{aligned}
& \mathrm{EI}_{\mathrm{i}} \mathrm{X}_{\mathrm{i}}{ }^{\prime \prime \prime}\left(\mathrm{x}_{\mathrm{i}}\right)-\mathrm{m}_{\mathrm{i}} \omega^{2} \mathrm{X}_{\mathrm{i}}\left(\mathrm{x}_{\mathrm{i}}\right)=0 \\
& \ddot{\mathrm{T}}(\mathrm{t})+2 \beta_{\mathrm{i}} \dot{\mathrm{T}}(\mathrm{t})+\omega^{2} \mathrm{~T}(\mathrm{t})=0
\end{aligned}
$$

where:

$$
\beta_{i}=\frac{\eta_{i}}{2 m_{i}}, \quad(i=1,2, \cdots, v)
$$

The solution of equation (3a) is given by the following relation:

$$
\begin{aligned}
\mathrm{X}_{\mathrm{i}}\left(\mathrm{x}_{\mathrm{i}}\right)=\mathrm{A}_{\mathrm{i}} \sin \mathrm{k}_{\mathrm{i}} \mathrm{x}_{\mathrm{i}}+\mathrm{B}_{\mathrm{i}} \cos \mathrm{k}_{\mathrm{i}} \mathrm{x}_{\mathrm{i}}+ \\
+\mathrm{C}_{\mathrm{i}} \sinh \mathrm{k}_{\mathrm{i}} \mathrm{x}_{\mathrm{i}}+\mathrm{D}_{\mathrm{i}} \cosh \mathrm{k}_{\mathrm{i}} \mathrm{x}_{\mathrm{i}}
\end{aligned}
$$

By setting:

$$
\begin{aligned}
& \mathrm{k}_{\mathrm{i}}^{4}=\frac{\mathrm{m}_{\mathrm{i}} \omega^{2}}{\mathrm{EI}_{\mathrm{i}}}, \quad \mathrm{c}_{\mathrm{i}}^{4}=\frac{\mathrm{m}_{\mathrm{i}} \mathrm{I}_{1}}{\mathrm{I}_{\mathrm{i}} \mathrm{m}_{1}}, \quad \mathrm{k}_{\mathrm{i}}=\mathrm{c}_{\mathrm{i}} \mathrm{k}, \\
& \mathrm{k}=\mathrm{k}_{1}, \quad \mathrm{~d}_{\mathrm{i}}=\frac{\mathrm{I}_{\mathrm{i}}}{\mathrm{I}_{1}} \quad(\text { for } \mathrm{i}=1,2, \cdots, v)
\end{aligned}
$$

eq(4) becomes:

$$
\begin{aligned}
\mathrm{X}_{\mathrm{i}}\left(\mathrm{x}_{\mathrm{i}}\right)=\mathrm{A}_{\mathrm{i}} \sin \mathrm{c}_{\mathrm{i}} \mathrm{kx}_{\mathrm{i}}+\mathrm{B}_{\mathrm{i}} \cos \mathrm{c}_{\mathrm{i}} \mathrm{kx}_{\mathrm{i}}+ \\
+\mathrm{C}_{\mathrm{i}} \sinh \mathrm{c}_{\mathrm{i}} \mathrm{kx}_{\mathrm{i}}+\mathrm{D}_{\mathrm{i}} \cosh \mathrm{c}_{\mathrm{i}} \mathrm{kx}_{\mathrm{i}}
\end{aligned}
$$
are:

The corresponding boundary conditions for each span $1^{\text {st }}$ span:

$$
\begin{aligned}
& X_{1}(0)=0, \quad X_{1}\left(\ell_{1}\right)=0, \quad X_{1}{ }^{\prime \prime}(0)=0, \\
& X_{1}{ }^{\prime \prime}\left(\ell_{1}\right)=d_{2} X_{2}{ }^{\prime \prime}(0), \quad X_{1}{ }^{\prime}\left(\ell_{1}\right)=X_{2}{ }^{\prime}(0) \\
& \quad i^{\text {th }} \text { span: } \\
& d_{i-1} X_{i-1}{ }^{\prime \prime}\left(\ell_{i-1}\right)=d_{i} X_{i}{ }^{\prime \prime}(0), \\
& X_{i-1}{ }^{\prime}\left(\ell_{i-1}\right)=X_{i}{ }^{\prime}(0), \quad X_{i}(0)=0, \\
& X_{i}\left(\ell_{i}\right)=0, \quad d_{i} X_{i}{ }^{\prime \prime}\left(\ell_{i}\right)=d_{i+1} X_{i+1}(0), \\
& X_{i}{ }^{\prime}\left(\ell_{i}\right)=X_{i+1}{ }^{\prime}(0) \\
& \quad v^{\text {th }} \text { span: } \\
& d_{v-1} X_{v-1}{ }^{\prime \prime}\left(\ell_{v-1}\right)=d_{v} X_{v}{ }^{\prime \prime}(0), \quad X_{v-1}{ }^{\prime}\left(\ell_{v-1}\right)=X_{v}{ }^{\prime}(0), \\
& X_{v}(0)=0, \quad X_{v}\left(\ell_{v}\right)=0, \quad X_{v}{ }^{\prime \prime}\left(\ell_{v}\right)=0
\end{aligned}
$$


The above conditions provide the following linear homogeneous system of equations:

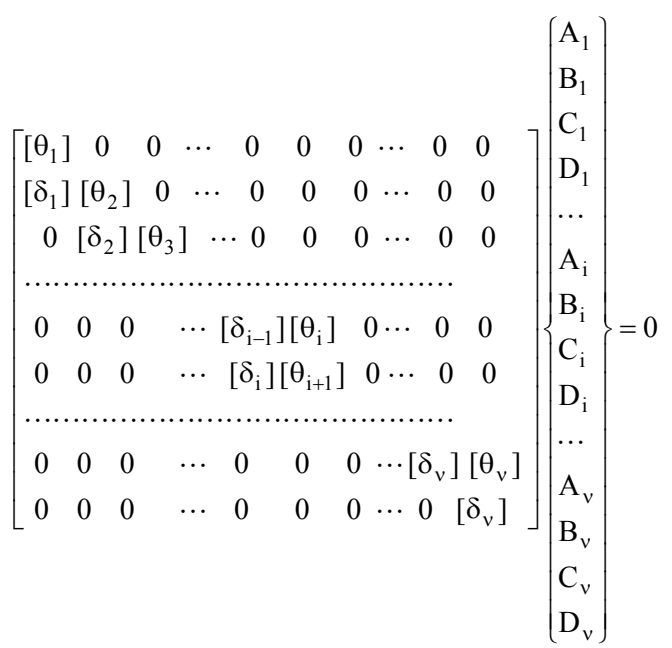

In order for the system to have non-trivial solutions, the determinant of the coefficients of the unknowns must be set equal to zero, thus the following condition must be satisfied:

$\left|\Delta_{v}\right|=0$

where the sub-matrices in eq(8) are:

$\left[\theta_{1}\right]=\left[\begin{array}{llll}0 & -1 & 0 & 1\end{array}\right]$

$\left[\delta_{1}\right]=\left[\begin{array}{cccc}0 & 1 & 0 & 1 \\ \sin \mathrm{k} \ell_{1} & \cos \ell_{1} & \sinh \ell_{1} & \cosh \ell_{1} \\ -\sin \mathrm{k} \ell_{1} & -\cos \ell_{1} & \sinh \ell_{1} & \cosh \ell_{1} \\ \cos \ell_{1} & -\sin \ell_{1} & \cosh \ell_{1} & \sinh \ell_{1}\end{array}\right]$

$\left[\theta_{2}\right]=\left[\begin{array}{cccc}0 & 0 & 0 & 0 \\ 0 & 0 & 0 & 0 \\ 0 & \mathrm{~d}_{2} \mathrm{c}_{2}^{2} & 0 & -\mathrm{d}_{2} \mathrm{c}_{2}^{2} \\ -\mathrm{c}_{2} & 0 & -\mathrm{c}_{2} & 0\end{array}\right]$

$\left[\delta_{\mathrm{i}}\right]=\left[\begin{array}{cccc}0 & 1 & 0 & 1 \\ \sin \mathrm{c}_{\mathrm{i}} \mathrm{k} \ell_{\mathrm{i}} & \cos \mathrm{c}_{\mathrm{i}} \mathrm{k} \ell_{\mathrm{i}} & \sinh \mathrm{c}_{\mathrm{i}} \mathrm{k} \ell_{\mathrm{i}} & \operatorname{coshc} \mathrm{c}_{\mathrm{i}} \mathrm{k} \ell_{\mathrm{i}} \\ -\mathrm{d}_{\mathrm{i}} \mathrm{c}_{\mathrm{i}}^{2} \sin \mathrm{c}_{\mathrm{i}} \mathrm{k} \ell_{\mathrm{i}} & -\mathrm{d}_{\mathrm{i}} \mathrm{c}_{\mathrm{i}}^{2} \cos \mathrm{c}_{\mathrm{i}} \mathrm{k} \ell_{\mathrm{i}} & \mathrm{d}_{\mathrm{i}} \mathrm{c}_{\mathrm{i}}^{2} \sinh \mathrm{c}_{\mathrm{i}} \mathrm{k} \ell_{\mathrm{i}} & \mathrm{d}_{\mathrm{i}} \mathrm{c}_{\mathrm{i}}^{2} \cosh \mathrm{c}_{\mathrm{i}} \mathrm{k} \ell_{\mathrm{i}} \\ \mathrm{c}_{\mathrm{i}} \cos \mathrm{c}_{\mathrm{i}} \mathrm{k} \ell_{\mathrm{i}} & -\mathrm{c}_{\mathrm{i}} \sin \mathrm{c}_{\mathrm{i}} \mathrm{k} \ell_{\mathrm{i}} & \mathrm{c}_{\mathrm{i}} \cosh \mathrm{c}_{\mathrm{i}} \mathrm{k} \ell_{\mathrm{i}} & \mathrm{c}_{\mathrm{i}} \sinh \mathrm{c}_{\mathrm{i}} \mathrm{k} \ell_{\mathrm{i}}\end{array}\right]$

$\left[\theta_{\mathrm{i}}\right]=\left[\begin{array}{cccc}0 & 0 & 0 & 0 \\ 0 & 0 & 0 & 0 \\ 0 & \mathrm{~d}_{\mathrm{i}} \mathrm{c}_{\mathrm{i}}^{2} & 0 & -\mathrm{d}_{\mathrm{i}} \mathrm{c}_{\mathrm{i}}^{2} \\ -\mathrm{c}_{\mathrm{i}} & 0 & -\mathrm{c}_{\mathrm{i}} & 0\end{array}\right]$

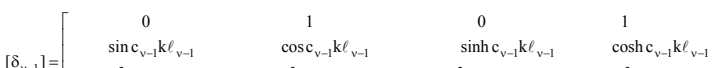

$\left[\delta_{v-1}\right]=\left[\begin{array}{cccc}-d_{v-1} c_{v-1}^{2} \sin c_{v-1} k \ell_{v-1} & -d_{v-1} c_{v-1}^{2} \cos c_{v-1} k \ell_{v-1} & d_{v-1} c_{v-1}^{2} \sinh c_{v-1} k \ell_{v-1} & d_{v-1} c_{v-1}^{2} \operatorname{coshc} c_{v-1} k \ell_{v-1} \\ c_{v-1} \cos c_{v-1} k \ell_{v-1} & -c_{v-1} \sin c_{v-1} k \ell_{v-1} & c_{v-1} \cosh c_{v-1} k \ell_{v-1} & c_{v-1} \sinh c_{v-1} k \ell_{v-1}\end{array}\right]$

$\left[\theta_{v}\right]=\left[\begin{array}{cccc}0 & 0 & 0 & 0 \\ 0 & 0 & 0 & 0 \\ 0 & \mathrm{~d}_{v} c_{v}^{2} & 0 & -\mathrm{d}_{v} \mathrm{c}_{v}^{2} \\ -\mathrm{c}_{v} & 0 & -\mathrm{c}_{v} & 0\end{array}\right]$ $\left[\delta_{v}\right]=\left[\begin{array}{cccc}0 & 1 & 0 & 1 \\ \sin \mathrm{c}_{v} \mathrm{k} \ell_{v} & \cos \mathrm{c}_{v} \mathrm{k} \ell_{v} & \sinh \mathrm{c}_{v} \mathrm{k} \ell_{v} & \cosh \mathrm{c}_{v} \mathrm{k} \ell_{v} \\ -\sin \mathrm{c}_{v} \mathrm{k} \ell_{v} & -\cos \mathrm{c}_{v} \mathrm{k} \ell_{v} & \sinh \mathrm{c}_{v} \mathrm{k} \ell_{v} & \cosh \mathrm{c}_{v} \mathrm{k} \ell_{v}\end{array}\right]$

(10h)

\section{Reduced Formulae for Eigenfrequencies}

Expanding the matrix of eq(9), we determine the following conversion formulae:

$$
\begin{aligned}
& \left|\Delta_{v}\right|=\mathrm{U}_{v} \cdot\left|\widetilde{\Delta}_{v-1}\right|+\mathrm{V}_{v} \cdot\left|\Delta_{v-1}\right| \\
& \left|\widetilde{\Delta}_{v}\right|=\mathrm{V}_{v} \cdot\left|\widetilde{\Delta}_{v-1}\right|+\mathrm{W}_{v} \cdot\left|\Delta_{v-1}\right|
\end{aligned}
$$

with:

$$
\begin{aligned}
& \mathrm{U}_{v}=4 \mathrm{~d}_{v} \mathrm{c}_{v-1} \mathrm{c}_{v}^{2} \cdot \sin \mathrm{c}_{v} \mathrm{k} \ell_{v} \cdot \sinh \mathrm{c}_{v} \mathrm{k} \ell_{v} \\
& \mathrm{~V}_{v}=2 \mathrm{~d}_{v-1} \mathrm{c}_{v-1}^{2} \mathrm{c}_{v}\left(\sin \mathrm{c}_{v} \mathrm{k} \ell_{v} \cdot \cosh \mathrm{c}_{v} \mathrm{k} \ell_{v}-\right. \\
& \left.\quad-\cos \mathrm{c}_{v} \mathrm{k} \ell_{v} \cdot \sinh \mathrm{c}_{v} \mathrm{k} \ell_{v}\right) \\
& \mathrm{W}_{v}=2 \mathrm{c}_{v-1} \mathrm{c}_{v}\left(1-\mathrm{d}_{v-1} \mathrm{c}_{v-1} \cos \mathrm{c}_{v} \mathrm{k} \ell_{v} \cdot \cosh \mathrm{c}_{v} \mathrm{k} \ell_{v}\right)
\end{aligned}
$$

where:

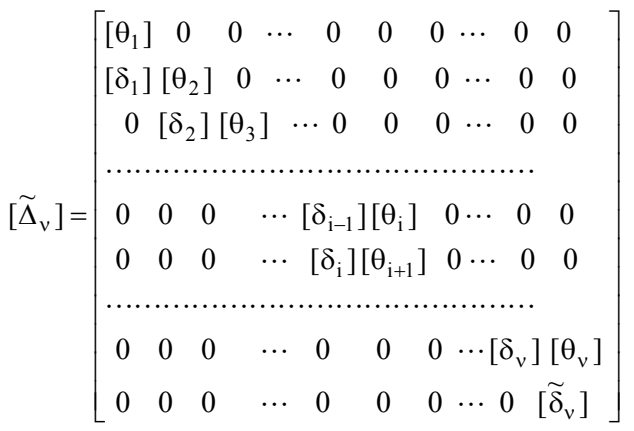

$\left[\widetilde{\delta}_{v}\right]=\left[\begin{array}{cccc}0 & 1 & 0 & 1 \\ \sin \mathrm{c}_{v} \mathrm{k} \ell_{v} & \cos \mathrm{c}_{v} \mathrm{k} \ell_{v} & \sinh \mathrm{c}_{v} \mathrm{k} \ell_{v} & \cosh \mathrm{c}_{v} \mathrm{k} \ell_{v} \\ \cos \mathrm{c}_{v} \mathrm{k} \ell_{v} & -\sin \mathrm{c}_{v} \mathrm{k} \ell_{v} & \cosh \mathrm{c}_{v} \mathrm{k} \ell_{v} & \sinh \mathrm{c}_{v} \mathrm{k} \ell_{v}\end{array}\right]$

$\left[\Delta_{1}\right]=4 \sin \mathrm{k} \ell_{1} \sinh \mathrm{k} \ell_{1}$

$\left[\widetilde{\Delta}_{1}\right]=2 \sin \mathrm{k} \ell_{1} \cosh \mathrm{k} \ell_{1}-2 \cos \mathrm{k} \ell_{1} \sinh \mathrm{k} \ell_{1}$

\section{Reduced Formulae for Shape Functions}

Let us consider now the equations related to the $\mathrm{i}^{\text {th }}$-span

$c_{i} A_{i}+c_{i} C_{i}=G_{i-1}$

$\mathrm{A}_{\mathrm{i}} \sin \mathrm{c}_{\mathrm{i}} \mathrm{k} \ell_{\mathrm{i}}+\mathrm{B}_{\mathrm{i}} \cos \mathrm{c}_{\mathrm{i}} \mathrm{k} \ell_{\mathrm{i}}+\mathrm{C}_{\mathrm{i}} \sinh \mathrm{c}_{\mathrm{i}} \mathrm{k} \ell_{\mathrm{i}}+\mathrm{D}_{\mathrm{i}} \cosh \mathrm{c}_{\mathrm{i}} \mathrm{k} \ell_{\mathrm{i}}=0$

$-\mathrm{d}_{\mathrm{i}} \mathrm{c}_{\mathrm{i}}^{2} \mathrm{~A}_{\mathrm{i}} \sin \mathrm{c}_{\mathrm{i}} \mathrm{k} \ell_{\mathrm{i}}-\mathrm{d}_{\mathrm{i}} \mathrm{c}_{\mathrm{i}}^{2} \mathrm{~B}_{\mathrm{i}} \cos \mathrm{c}_{\mathrm{i}} \mathrm{k} \ell_{\mathrm{i}}+\mathrm{d}_{\mathrm{i}} \mathrm{c}_{\mathrm{i}}^{2} \mathrm{C}_{\mathrm{i}} \sinh \mathrm{c}_{\mathrm{i}} \mathrm{k} \ell_{\mathrm{i}}+$ $+\mathrm{d}_{\mathrm{i}} \mathrm{c}_{\mathrm{i}}^{2} \mathrm{D}_{\mathrm{i}} \cosh \mathrm{c}_{\mathrm{i}} \mathrm{k} \ell_{\mathrm{i}}=\mathrm{d}_{\mathrm{i}+1} \mathrm{c}_{\mathrm{i}+1}^{2}\left(\mathrm{D}_{\mathrm{i}+1}-\mathrm{B}_{\mathrm{i}+1}\right)$

$\mathrm{B}_{\mathrm{i}}+\mathrm{D}_{\mathrm{i}}=0$

for $i=2$ to $(v-1)$, and $G_{i}$ is given by

$\mathrm{G}_{\mathrm{i}}=\left(\mathrm{A}_{\mathrm{i}} \cos \mathrm{c}_{\mathrm{i}} \mathrm{k} \ell_{\mathrm{i}}-\mathrm{B}_{\mathrm{i}} \sin \mathrm{c}_{\mathrm{i}} \mathrm{k} \ell_{\mathrm{i}}+\mathrm{C}_{\mathrm{i}} \cosh \mathrm{c}_{\mathrm{i}} \mathrm{k} \ell_{\mathrm{i}}+\mathrm{D}_{\mathrm{i}} \sinh \mathrm{c}_{\mathrm{i}} \mathrm{k} \ell_{\mathrm{i}}\right) \mathrm{c}_{\mathrm{i}}$

Because of eq(13d), that is valid also for (i-1), the above system can be written as:

$\mathrm{c}_{\mathrm{i}} \mathrm{A}_{\mathrm{i}}+\mathrm{c}_{\mathrm{i}} \mathrm{C}_{\mathrm{i}}=\mathrm{G}_{\mathrm{i}-1}$ 
$\mathrm{A}_{\mathrm{i}} \sin \mathrm{c}_{\mathrm{i}} \mathrm{k} \ell_{\mathrm{i}}+\mathrm{B}_{\mathrm{i}} \cos \mathrm{c}_{\mathrm{i}} \mathrm{k} \ell_{\mathrm{i}}+\mathrm{C}_{\mathrm{i}} \sinh \mathrm{c}_{\mathrm{i}} \mathrm{k} \ell_{\mathrm{i}}+\mathrm{D}_{\mathrm{i}} \cosh \mathrm{c}_{\mathrm{i}} \mathrm{k} \ell_{\mathrm{i}}=0$

$-\mathrm{d}_{\mathrm{i}} \mathrm{c}_{\mathrm{i}}^{2} \mathrm{~A}_{\mathrm{i}} \sin \mathrm{c}_{\mathrm{i}} \mathrm{k} \ell_{\mathrm{i}}-\mathrm{d}_{\mathrm{i}} \mathrm{c}_{\mathrm{i}}^{2} \mathrm{~B}_{\mathrm{i}} \cos \mathrm{c}_{\mathrm{i}} \mathrm{k} \ell_{\mathrm{i}}+\mathrm{d}_{\mathrm{i}} \mathrm{c}_{\mathrm{i}}^{2} \mathrm{C}_{\mathrm{i}} \sinh \mathrm{c}_{\mathrm{i}} \mathrm{k} \ell_{\mathrm{i}}+$

$$
+d_{i} c_{i}^{2} D_{i} \cosh c_{i} k \ell_{i}=-2 d_{i+1} c_{i+1}^{2} B_{i+1}
$$

$\mathrm{B}_{\mathrm{i}}+\mathrm{D}_{\mathrm{i}}=0$

which has the following solution:

$$
\begin{aligned}
& A_{i}=a_{i} G_{i-1}+\widetilde{a}_{i} B_{i+1} \\
& B_{i}=b_{i} G_{i-1}+\widetilde{b}_{i} B_{i+1} \\
& C_{i}=\gamma_{i} G_{i-1}+\widetilde{\gamma}_{i} B_{i+1} \\
& D_{i}=\delta_{i} G_{i-1}+\widetilde{\delta}_{i} B_{i+1}
\end{aligned}
$$

where:

$$
\begin{aligned}
& \mathrm{a}_{\mathrm{i}}=\frac{\cos \mathrm{c}_{\mathrm{i}} \mathrm{k} \ell_{\mathrm{i}} \sinh \mathrm{c}_{\mathrm{i}} \mathrm{k} \ell_{\mathrm{i}}}{\mathrm{c}_{\mathrm{i}} \Lambda_{\mathrm{i}}} \\
& \widetilde{\mathrm{a}}_{\mathrm{i}}=\frac{-\mathrm{d}_{\mathrm{i}+1} \mathrm{c}_{\mathrm{i}+1}^{2}\left(\cosh \mathrm{c}_{\mathrm{i}} \mathrm{k} \ell_{\mathrm{i}}-\cos \mathrm{c}_{\mathrm{i}} \mathrm{k} \ell_{\mathrm{i}}\right)}{\mathrm{d}_{\mathrm{i}} \mathrm{c}_{\mathrm{i}}^{2} \Lambda_{\mathrm{i}}} \\
& \mathrm{b}_{\mathrm{i}}=\frac{-\sin \mathrm{c}_{\mathrm{i}} \mathrm{k} \ell_{\mathrm{i}} \sinh \mathrm{c}_{\mathrm{i}} \mathrm{k} \ell_{\mathrm{i}}}{\mathrm{c}_{\mathrm{i}} \Lambda_{\mathrm{i}}} \\
& \widetilde{\mathrm{b}}_{\mathrm{i}}=\frac{\mathrm{d}_{\mathrm{i}+1} \mathrm{c}_{\mathrm{i}+1}^{2}\left(\sinh \mathrm{c}_{\mathrm{i}} \mathrm{k} \ell_{\mathrm{i}}-\sin \mathrm{c}_{\mathrm{i}} \mathrm{k} \ell_{\mathrm{i}}\right)}{\mathrm{d}_{\mathrm{i}} \mathrm{c}_{\mathrm{i}}^{2} \Lambda_{\mathrm{i}}} \\
& \gamma_{\mathrm{i}}=\frac{-\sin \mathrm{c}_{\mathrm{i}} \mathrm{k} \ell_{\mathrm{i}} \cosh \mathrm{c}_{\mathrm{i}} \mathrm{k} \ell_{\mathrm{i}}}{\mathrm{c}_{\mathrm{i}} \Lambda_{\mathrm{i}}} \\
& \widetilde{\gamma}_{\mathrm{i}}=\frac{\mathrm{d}_{\mathrm{i}+1} \mathrm{c}_{\mathrm{i}+1}^{2}\left(\cosh \mathrm{c}_{\mathrm{i}} \mathrm{k} \ell_{\mathrm{i}}-\cos \mathrm{c}_{\mathrm{i}} \mathrm{k} \ell_{\mathrm{i}}\right)}{\mathrm{d}_{\mathrm{i}} \mathrm{c}_{\mathrm{i}}^{2} \Lambda_{\mathrm{i}}} \\
& \tilde{\delta}_{\mathrm{i}}=\frac{\sin \mathrm{c}_{\mathrm{i}} \mathrm{k} \ell_{\mathrm{i}} \sinh \mathrm{c}_{\mathrm{i}} \mathrm{k} \ell_{\mathrm{i}}}{\mathrm{c}_{\mathrm{i}} \Lambda_{\mathrm{i}}} \\
& \widetilde{\mathrm{d}}_{\mathrm{i}}=\frac{\cos \mathrm{c}_{\mathrm{i}} \mathrm{k} \ell_{\mathrm{i}} \sinh \mathrm{c}_{\mathrm{i}} \mathrm{k} \ell_{\mathrm{i}}-\sin \mathrm{c}_{\mathrm{i}} \mathrm{k} \ell_{\mathrm{i}} \cosh \mathrm{c}_{\mathrm{i}} \mathrm{k} \ell_{\mathrm{i}}}{\left.\sinh \mathrm{c}_{\mathrm{i}} \mathrm{k} \ell_{\mathrm{i}}-\sin \mathrm{c}_{\mathrm{i}} \mathrm{k} \ell_{\mathrm{i}}\right)}
\end{aligned}
$$

We have now to determine the conversion formulae for $\mathrm{G}_{\mathrm{i}}$. From the system

$\mathrm{B}_{1}=\mathrm{D}_{1}=0$

$\mathrm{A}_{1} \sin \mathrm{k} \ell_{1}+\mathrm{C}_{1} \sinh \mathrm{k} \ell_{1}=0$

$-\mathrm{A}_{1} \sin \mathrm{k} \ell_{1}+\mathrm{C}_{1} \sinh \mathrm{k} \ell_{1}=-2 \mathrm{~d}_{2} \mathrm{c}_{2}^{2} \mathrm{~B}_{2}$

we find that:

$$
\begin{aligned}
\mathrm{A}_{1} & =\frac{\mathrm{d}_{2} \mathrm{c}_{2}^{2}}{\sin \mathrm{k} \ell_{1}} \mathrm{~B}_{2} \\
\mathrm{C}_{1} & =-\frac{\mathrm{d}_{2} \mathrm{c}_{2}^{2}}{\sinh \mathrm{k} \ell_{1}} \mathrm{~B}_{2} \\
\mathrm{G}_{1} & =\mathrm{d}_{2} \mathrm{c}_{2}^{2}\left(\cot \mathrm{k} \ell_{1}-\operatorname{coth} \mathrm{k} \ell_{1}\right) \mathrm{B}_{2}=\varepsilon_{1} \mathrm{~B}_{2}
\end{aligned}
$$

where:

$\varepsilon_{1}=\mathrm{d}_{2} \mathrm{c}_{2}^{2}\left(\cot \mathrm{k} \ell_{1}-\operatorname{coth} \mathrm{k} \ell_{1}\right)$

In general, we set:

$\mathrm{G}_{\mathrm{i}}=\varepsilon_{\mathrm{i}} \mathrm{B}_{\mathrm{i}+1}$

Then, because of eq(16b) we get:

$\mathrm{B}_{\mathrm{i}}=\mathrm{b}_{\mathrm{i}} \cdot \varepsilon_{\mathrm{i}-1} \cdot \mathrm{B}_{\mathrm{i}}+\tilde{\mathrm{b}}_{\mathrm{i}} \cdot \mathrm{B}_{\mathrm{i}+1}$

and thus

$\mathrm{B}_{\mathrm{i}}=\frac{\tilde{\mathrm{b}}_{\mathrm{i}}}{1-\mathrm{b}_{\mathrm{i}} \cdot \varepsilon_{\mathrm{i}-1}} \mathrm{~B}_{\mathrm{i}+1}$

or finally

$\mathrm{B}_{\mathrm{i}}=\zeta_{\mathrm{i}} \cdot \mathrm{B}_{\mathrm{i}+1}$

where

$$
\zeta_{\mathrm{i}}=\frac{\tilde{\mathrm{b}}_{\mathrm{i}}}{1-\mathrm{b}_{\mathrm{i}} \cdot \varepsilon_{\mathrm{i}-1}}
$$

Therefore, eqs(16) are written:

$$
\begin{aligned}
& \mathrm{A}_{\mathrm{i}}=\left(\mathrm{a}_{\mathrm{i}} \cdot \varepsilon_{\mathrm{i}-1} \cdot \zeta_{\mathrm{i}}+\widetilde{\mathrm{a}}_{\mathrm{i}}\right) \mathrm{B}_{\mathrm{i}+1} \\
& \mathrm{~B}_{\mathrm{i}}=\left(\mathrm{b}_{\mathrm{i}} \cdot \varepsilon_{\mathrm{i}-1} \cdot \zeta_{\mathrm{i}}+\widetilde{\mathrm{b}}_{\mathrm{i}}\right) \mathrm{B}_{\mathrm{i}+1} \\
& \mathrm{C}_{\mathrm{i}}=\left(\gamma_{\mathrm{i}} \cdot \varepsilon_{\mathrm{i}-1} \cdot \zeta_{\mathrm{i}}+\widetilde{\gamma}_{\mathrm{i}}\right) \mathrm{B}_{\mathrm{i}+1} \\
& \mathrm{D}_{\mathrm{i}}=\left(\delta_{\mathrm{i}} \cdot \varepsilon_{\mathrm{i}-1} \cdot \zeta_{\mathrm{i}}+\widetilde{\delta}_{\mathrm{i}}\right) \mathrm{B}_{\mathrm{i}+1}
\end{aligned}
$$

Introducing eqs(21) into eqs(14), we get:

$$
\begin{aligned}
\mathrm{G}_{\mathrm{i}}= & {\left[\mathrm{c}_{\mathrm{i}}\left(\mathrm{a}_{\mathrm{i}} \varepsilon_{\mathrm{i}-1} \zeta_{\mathrm{i}}+\widetilde{\mathrm{a}}_{\mathrm{i}}\right) \cos \mathrm{c}_{\mathrm{i}} \mathrm{k} \ell_{\mathrm{i}}-\mathrm{c}_{\mathrm{i}}\left(\mathrm{b}_{\mathrm{i}} \varepsilon_{\mathrm{i}-1} \zeta_{\mathrm{i}}+\widetilde{\mathrm{b}}_{\mathrm{i}}\right) \sin \mathrm{c}_{\mathrm{i}} \mathrm{k} \ell_{\mathrm{i}}+\right.} \\
& \left.\mathrm{c}_{\mathrm{i}}\left(\gamma_{\mathrm{i}} \varepsilon_{\mathrm{i}-1} \zeta_{\mathrm{i}}+\widetilde{\gamma}_{\mathrm{i}}\right) \cosh \mathrm{c}_{\mathrm{i}} \mathrm{k} \ell_{\mathrm{i}}+\mathrm{c}_{\mathrm{i}}\left(\delta_{\mathrm{i}} \varepsilon_{\mathrm{i}-1} \zeta_{\mathrm{i}}+\widetilde{\delta}_{\mathrm{i}}\right) \sinh \mathrm{c}_{\mathrm{i}} \mathrm{k} \ell_{\mathrm{i}}\right] \cdot \mathrm{B}_{\mathrm{i}+1}
\end{aligned}
$$

and:

$$
\begin{aligned}
\varepsilon_{\mathrm{i}}= & \mathrm{c}_{\mathrm{i}}\left(\mathrm{a}_{\mathrm{i}} \varepsilon_{\mathrm{i}-1} \zeta_{\mathrm{i}}+\widetilde{\mathrm{a}}_{\mathrm{i}}\right) \cos \mathrm{c}_{\mathrm{i}} \mathrm{k} \ell_{\mathrm{i}}-\mathrm{c}_{\mathrm{i}}\left(\mathrm{b}_{\mathrm{i}} \varepsilon_{\mathrm{i}-1} \zeta_{\mathrm{i}}+\widetilde{\mathrm{b}}_{\mathrm{i}}\right) \sin \mathrm{c}_{\mathrm{i}} \mathrm{k} \ell_{\mathrm{i}}+ \\
& \mathrm{c}_{\mathrm{i}}\left(\gamma_{\mathrm{i}} \varepsilon_{\mathrm{i}-1} \zeta_{\mathrm{i}}+\widetilde{\gamma}_{\mathrm{i}}\right) \cosh \mathrm{c}_{\mathrm{i}} \mathrm{k} \ell_{\mathrm{i}}+\mathrm{c}_{\mathrm{i}}\left(\delta_{\mathrm{i}} \varepsilon_{\mathrm{i}-1} \zeta_{\mathrm{i}}+\widetilde{\delta}_{\mathrm{i}}\right) \sinh \mathrm{c}_{\mathrm{i}} \mathrm{k} \ell_{\mathrm{i}}
\end{aligned}
$$

with

$\varepsilon_{1}=\mathrm{d}_{2} \mathrm{c}_{2}^{2}\left(\cot \mathrm{k} \ell_{1}-\operatorname{coth} \mathrm{k} \ell_{1}\right)$

Finally, from the system

$\mathrm{B}_{v}+\mathrm{D}_{v}=0$

$\mathrm{A}_{v} \sin \mathrm{c}_{\mathrm{v}} \mathrm{k} \ell_{v}+\mathrm{B}_{v} \cos \mathrm{c}_{v} \mathrm{k} \ell_{v}+\mathrm{C}_{v} \sinh \mathrm{c}_{v} \mathrm{k} \ell_{v}+\mathrm{D}_{v} \cosh \mathrm{c}_{v} \mathrm{k} \ell_{v}=0$

$-\mathrm{A}_{v} \sin \mathrm{c}_{v} \mathrm{k} \ell_{v}-\mathrm{B}_{v} \cos \mathrm{c}_{v} \mathrm{k} \ell_{v}+\mathrm{C}_{v} \sinh \mathrm{c}_{v} \mathrm{k} \ell_{v}+\mathrm{D}_{v} \cosh \mathrm{c}_{v} \mathrm{k} \ell_{v}=0$

we determine the coefficients $\mathrm{A}_{v}$ and $\mathrm{B}_{v}$ as follows:

$$
\begin{aligned}
& \mathrm{A}_{v}=-\mathrm{B}_{v} \cdot \cot \mathrm{c}_{v} \mathrm{k} \ell_{v} \\
& \mathrm{C}_{v}=\mathrm{B}_{v} \cdot \operatorname{coth} \mathrm{c}_{v} \mathrm{k} \ell_{v} \\
& \mathrm{D}_{v}=-\mathrm{B}_{v}
\end{aligned}
$$

Hence, every coefficient is expressed as a function of $\mathrm{B}_{v}$. Constant $B_{v}$ is a random number that can take any value. Usually, we set $\mathrm{B}_{v}=1$. Thus, we get the following relations:

Span 1: 


$$
\begin{aligned}
& \mathrm{X}_{1}=\mathrm{d}_{2} \mathrm{c}_{2}^{2} \zeta_{2} \zeta_{3} \cdots \zeta_{v-1}\left(\frac{1}{\sin \mathrm{k} \ell_{1}} \sin \mathrm{kx}_{1}-\frac{1}{\sinh \mathrm{k} \ell_{1}} \sinh \mathrm{kx}_{1}\right)(25 \mathrm{a}) \\
& \quad \text { Span i (for } \mathrm{i}=2 \text { to } v-1): \\
& \mathrm{X}_{\mathrm{i}}=\zeta_{\mathrm{i}+1} \zeta_{\mathrm{i}+2} \cdots \zeta_{v-1}\left[\left(\mathrm{a}_{\mathrm{i}} \varepsilon_{\mathrm{i}-1} \zeta_{\mathrm{i}}+\widetilde{\mathrm{a}}_{\mathrm{i}}\right) \sin \mathrm{c}_{\mathrm{i}} \mathrm{k} \ell_{\mathrm{i}}+\right. \\
& +\left(\mathrm{b}_{\mathrm{i}} \varepsilon_{\mathrm{i}-1} \zeta_{\mathrm{i}}+\widetilde{\mathrm{b}}_{\mathrm{i}}\right) \cos \mathrm{c}_{\mathrm{i}} \mathrm{k} \ell_{\mathrm{i}}+\left(\gamma_{\mathrm{i}} \varepsilon_{\mathrm{i}-1} \zeta_{\mathrm{i}}+\widetilde{\gamma}_{\mathrm{i}}\right) \sinh \mathrm{c}_{\mathrm{i}} \mathrm{k} \ell_{\mathrm{i}}+(25 \mathrm{~b}) \\
& \left.+\left(\delta_{\mathrm{i}} \varepsilon_{\mathrm{i}-1} \zeta_{\mathrm{i}}+\widetilde{\delta}_{\mathrm{i}}\right) \cosh \mathrm{c}_{\mathrm{i}} \mathrm{k} \ell_{\mathrm{i}}\right]
\end{aligned}
$$

Span $v$ :

$$
\begin{aligned}
\mathrm{X}_{\mathrm{v}}=- & \cot \mathrm{c}_{\mathrm{v}} \mathrm{k} \ell_{\mathrm{v}} \sin \mathrm{c}_{\mathrm{v}} \mathrm{kx}_{\mathrm{v}}+\cos \mathrm{c}_{\mathrm{v}} \mathrm{kx}_{\mathrm{v}}+ \\
& +\operatorname{coth}_{\mathrm{v}} \mathrm{k} \ell_{\mathrm{v}} \cdot \sinh \mathrm{c}_{\mathrm{v}} \mathrm{kx}_{\mathrm{v}}-\cos _{\mathrm{v}} \mathrm{kx}_{\mathrm{v}}
\end{aligned}
$$

\section{Orthogonality Conditions}

From eq(3a), we consider firstly the mode $n$ of the $i^{\text {th }}$ span, which we multiply by $\mathrm{X}_{\mathrm{ik}}$. We next consider the mode $\mathrm{k}$ of the $\mathrm{i}^{\text {th }}$ span, which we multiply by $\mathrm{X}_{\text {in. }}$. Integrating the outcome from 0 to $\ell_{\mathrm{i}}$ taking into account the boundary conditions and adding the results, we determine the following orthogonality conditions:

$$
\sum_{\mathrm{i}=1}^{v}\left(\mathrm{~m}_{\mathrm{i}} \int_{0}^{\ell_{\mathrm{i}}} \mathrm{X}_{\mathrm{in}} \mathrm{X}_{\mathrm{ik}} \mathrm{dx}\right)=0 \quad(\text { for } \mathrm{n} \neq \mathrm{k})
$$

\section{The Forced Vibration of Continuous Beams}

The complete equation of motion of a beam under the action of a moving mass $\mathrm{M}$ is:

$$
\begin{aligned}
\operatorname{EIw}^{\prime \prime \prime}(\mathrm{x}, \mathrm{t})+\mathrm{cw}(\mathrm{x}, \mathrm{t})+\mathrm{m} \ddot{w}(\mathrm{x}, \mathrm{t}) & = \\
& =(\mathrm{Mg}-\mathrm{M} \ddot{\mathrm{w}}) \cdot \delta(\mathrm{x}-\mathrm{v} \mathrm{t})
\end{aligned}
$$

Given that the influence of the mass $\mathrm{M}$ of a moving load is negligible [17] for values,

$$
\mathrm{M} / \sum_{\mathrm{i}=1}^{v} \mathrm{~m}_{\mathrm{i}} \ell_{\mathrm{i}}<0.3
$$

we will study the continuous beam crossed by a moving force with constant speed.

Let us consider now a load $\mathrm{P}=\mathrm{Mg}$ crossing the bridge with a constant velocity $v$. The load enters the beam from its left end at the time instant $\mathrm{t}=0$. When the load crosses the $\mathrm{i}^{\text {th }}$ span, in time interval $\sum_{1} \frac{\ell_{\mathrm{i}}}{\mathrm{v}} \leq \mathrm{t} \leq \sum_{1} \frac{\ell_{\mathrm{i}}}{\mathrm{v}}$, the following equa-
tions are valid:

$$
\begin{aligned}
& \mathrm{EI}_{1} \mathrm{w}_{1}{ }^{\prime \prime \prime}\left(\mathrm{x}_{1}, \mathrm{t}_{\mathrm{i}}\right)+\mathrm{cw}_{1}\left(\mathrm{x}_{1}, \mathrm{t}_{\mathrm{i}}\right)+\mathrm{m}_{1} \ddot{\mathrm{w}}_{1}\left(\mathrm{x}_{1}, \mathrm{t}_{\mathrm{i}}\right)=0 \\
& E I_{i-1} w_{i-1} " '\left(x_{i-1}, t_{i}\right)+c w_{i-1}\left(x_{i-1}, t_{i}\right)+m_{i-1} \ddot{w}_{i-1}\left(x_{i-1}, t_{i}\right)=0 \\
& E I_{1} w_{i}{ }^{\prime \prime \prime}\left(x_{i}, t_{i}\right)+w_{i}\left(x_{i}, t_{i}\right)+m_{i} \ddot{w}_{i}\left(x_{i}, t_{i}\right)=P \cdot \delta\left(x_{i}-v t_{i}\right) \\
& \mathrm{EI}_{\mathrm{i}+1} \mathrm{w}_{\mathrm{i}+1}{ }^{\prime \prime \prime}\left(\mathrm{x}_{\mathrm{i}+1}, \mathrm{t}_{\mathrm{i}}\right)+\mathrm{cw}_{\mathrm{i}+1}\left(\mathrm{x}_{\mathrm{i}+1}, \mathrm{t}_{\mathrm{i}}\right)+\mathrm{m}_{\mathrm{i}+1} \ddot{\mathrm{w}}_{\mathrm{i}+1}\left(\mathrm{x}_{\mathrm{i}+1}, \mathrm{t}_{\mathrm{i}}\right)=0 \\
& \mathrm{EI}_{v} \mathrm{w}_{v}{ }^{\prime \prime \prime}\left(\mathrm{x}_{v}, \mathrm{t}_{\mathrm{i}}\right)+\mathrm{cw}_{\mathrm{v}}\left(\mathrm{x}_{v}, \mathrm{t}_{\mathrm{i}}\right)+\mathrm{m}_{\mathrm{v}} \ddot{\mathrm{w}}_{\mathrm{v}}\left(\mathrm{x}_{v}, \mathrm{t}_{\mathrm{i}}\right)=0
\end{aligned}
$$

where:

$$
\mathrm{t}_{\mathrm{i}}=\mathrm{t}-\sum_{1}^{\mathrm{i}-1} \frac{\ell_{\mathrm{i}}}{\mathrm{v}}
$$

and $\delta$ is the Dirac unit function.

We are searching for a solution under the form:

$$
\mathrm{w}_{\mathrm{i}}\left(\mathrm{x}_{\mathrm{i}}, \mathrm{t}\right)=\sum_{\mathrm{n}}\left(\mathrm{X}_{\mathrm{in}} \mathrm{T}_{\mathrm{n}}\right) \quad(\mathrm{i}=1,2, \cdots, v)
$$

where the index $\mathrm{n}$ shows the number of the used eigenmodes.

Thus, eqs(27) become:

$$
\begin{aligned}
& E I_{j} \sum X_{n=1}^{\prime \prime \prime} T_{i n}+c \sum_{n} X_{j n} \dot{T}_{i n}+m_{j} \sum X_{n j n} \ddot{T}_{i n}=0 \quad \text { for } j \neq i \\
& E I_{j} \sum_{n} X_{j n}^{\prime \prime \prime \prime} T_{i n}+c \sum_{n} X_{j n} \dot{T}_{i n}+m_{j} \sum X_{n} X_{j n} \ddot{i n}_{i n}=P \delta\left(x_{j}-v t_{i}\right) \quad \text { for } j=i
\end{aligned}
$$

and $\mathrm{j}=1,2, \ldots, v$.

Taking into account that eq(3a) is valid, eq(29) become:

$$
\begin{array}{ll}
m_{j} \sum \omega_{n}^{2} X_{j n} T_{i n}+c \sum_{n} X_{j n} \dot{T}_{i n}+m_{j} \sum X_{n} X_{j n} \ddot{T}_{i n}=0 & \text { for } j \neq i \\
m_{j} \sum \omega_{n}^{2} X_{j n} T_{i n}+c \sum_{n} X_{j n} \dot{T}_{i n}+m_{j} \sum X_{n} X_{j n} \ddot{T}_{i n}=P \delta\left(x_{j}-v t_{i}\right) & \text { for } j=i
\end{array}
$$

Multiplying the corresponding equation $\mathrm{j}$ by $\mathrm{X}_{\mathrm{jk}}$, integrating the outcome from 0 to $\ell_{\mathrm{j}}$, adding the resulting expressions and using the orthogonality conditions, we get:

$$
\ddot{\mathrm{T}}_{\mathrm{in}}+2 \beta_{\mathrm{i}} \dot{\mathrm{T}}_{\mathrm{in}}+\omega_{\mathrm{n}}^{2} \mathrm{~T}_{\mathrm{in}}=\frac{\mathrm{P}}{\sum_{v}\left(\mathrm{~m}_{\mathrm{i}} \int_{0}^{\ell_{\mathrm{i}}} \mathrm{X}_{\mathrm{in}}^{2} \mathrm{dx}\right)} \cdot \mathrm{X}_{\mathrm{in}}\left(\mathrm{vt}_{\mathrm{i}}\right)
$$

with

$$
\mathrm{t}_{\mathrm{i}}=\mathrm{t}-\sum_{1}^{\mathrm{i}-1} \frac{\ell_{\mathrm{i}}}{\mathrm{v}}
$$

The solution of eq(30a) is given by the Duhamel's integral:

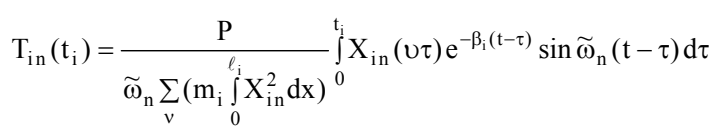

where:

$$
\widetilde{\omega}_{\mathrm{n}}=\sqrt{\omega_{\mathrm{n}}^{2}-\beta_{\mathrm{i}}^{2}}
$$

\section{Numerical Examples and Discussion}

Let us consider the multi-span beam of Fig. (1) made from a homogeneous, isotropic material with Young's modulus $\mathrm{E}=2.1 \times 10^{10} \mathrm{kN} / \mathrm{m}^{2}$.

The beam with total length $\ell=138 \mathrm{~m}$ consists of five spans with lengths $\ell_{1}=24 \mathrm{~m}, \ell_{2}=\ell_{3}=\ell_{4}=30 \mathrm{~m}$, and $\ell_{5}=24 \mathrm{~m}$, respectively, moment of inertia $\mathrm{I}_{\mathrm{b}}=0.05 \mathrm{~m}^{4}$, mass per unit length $\mathrm{m}_{\mathrm{b}}=500 \mathrm{~kg} / \mathrm{m}$ and damping parameter $\beta=0.02$. Since the beam properties are constant all over the length of the beam, the parameters $\mathrm{c}_{\mathrm{i}}$, and $\mathrm{d}_{\mathrm{i}}$ in eq(5) are $\mathrm{c}_{\mathrm{i}}=\mathrm{d}_{\mathrm{i}}=1$ and by formulating and solving the eigenfrequency equation (9), the first five eigenfrequencies are determined: $\omega_{1}=17.5324$, $\omega_{2}=21.9206, \omega_{3}=28.0297, \omega_{4}=34.6396, \omega_{5}=37.1087$. Then, the corresponding eigenshapes are obtained using the relations $(25 \mathrm{a}, \mathrm{b}, \mathrm{c})$. In Figs. (2 to 6) one can see in graphical form the first five eigenshapes for free vibration of the 5-span beam.

In the following Figs. (7, 8 and $\mathbf{9}$ ) one can see the dynamic influence lines referring to the midpoints of the first three spans, i.e. $w_{1}\left(\ell_{1} / 2, t\right), w_{2}\left(\ell_{2} / 2, t\right)$ and $w_{3}\left(\ell_{3} / 2, t\right)$. The 


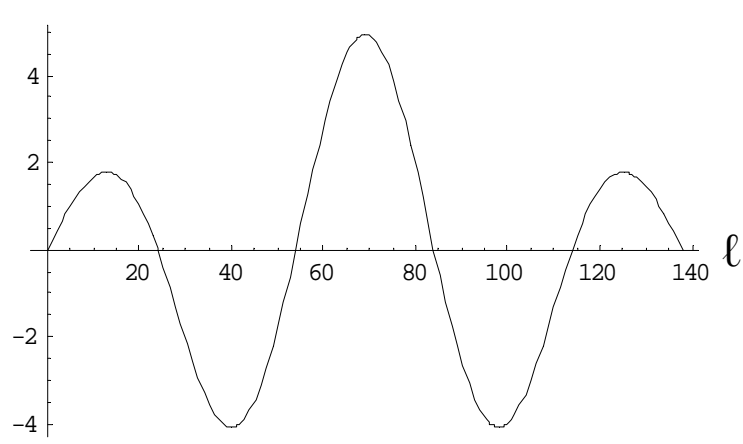

Fig. (2). The fundamental eigenshape of the free vibrating beam $\left(\omega_{1}=17.5324 \mathrm{sec}^{-1}\right)$.

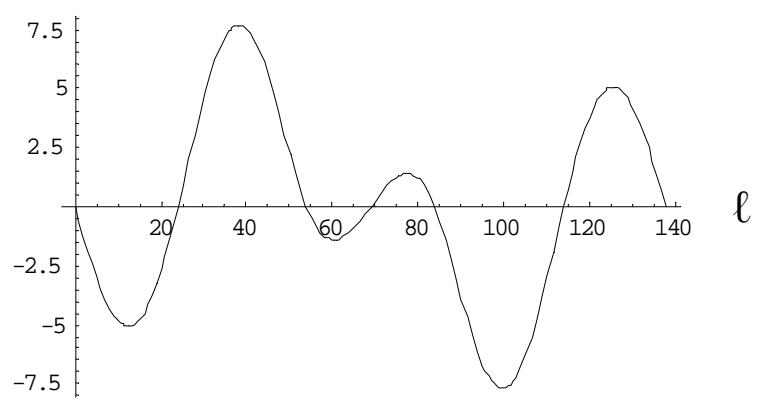

Fig. (3). The second eigenshape of the free vibrating beam $\left(\omega_{2}=21.9206 \mathrm{sec}^{-1}\right)$.

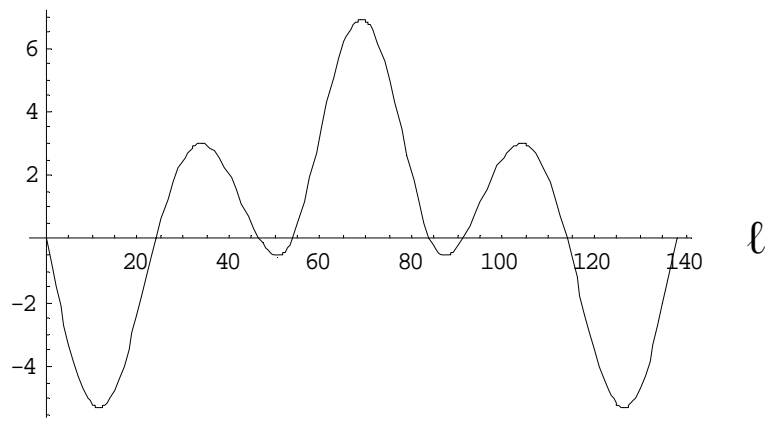

Fig. (4). The third eigenshape of the free vibrating beam $\left(\omega_{3}=28.0297 \mathrm{sec}^{-1}\right)$.

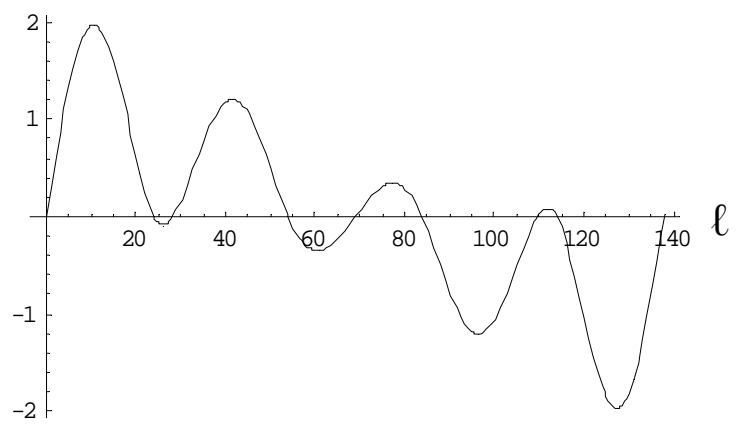

Fig. (5). The fourth eigenshape of the free vibrating beam $\left(\omega_{4}=34.6396 \mathrm{sec}^{-1}\right)$.

continuous influence line refers to velocity $v=20 \mathrm{~m} / \mathrm{s}$, while the dotted influence line refers to velocity $\mathrm{v}=40 \mathrm{~m} / \mathrm{s}$.

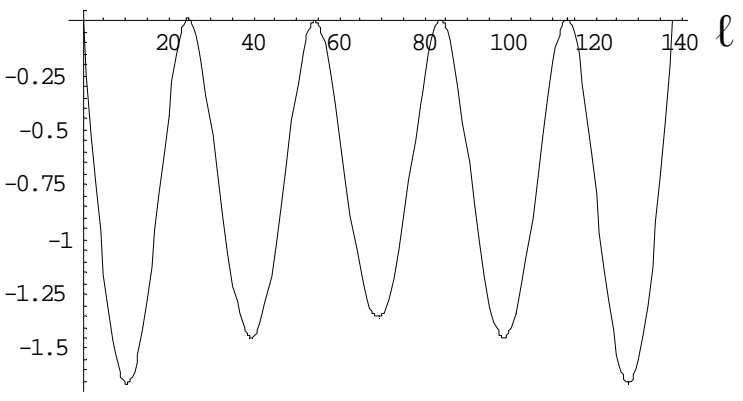

Fig. (6). The fifth eigenshape of the free vibrating beam $\left(\omega_{5}=37.1087 \mathrm{sec}^{-1}\right)$.

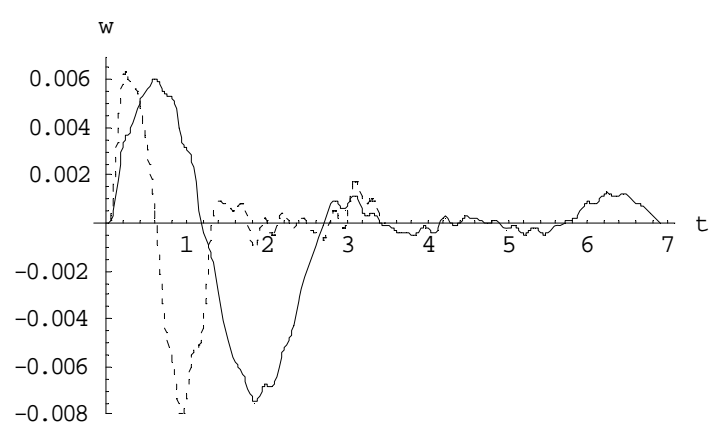

Fig. (7). Influence lines at $\mathrm{w}_{1}\left(\ell_{1} / 2, \mathrm{t}\right)$ for forced vibration of the beam.

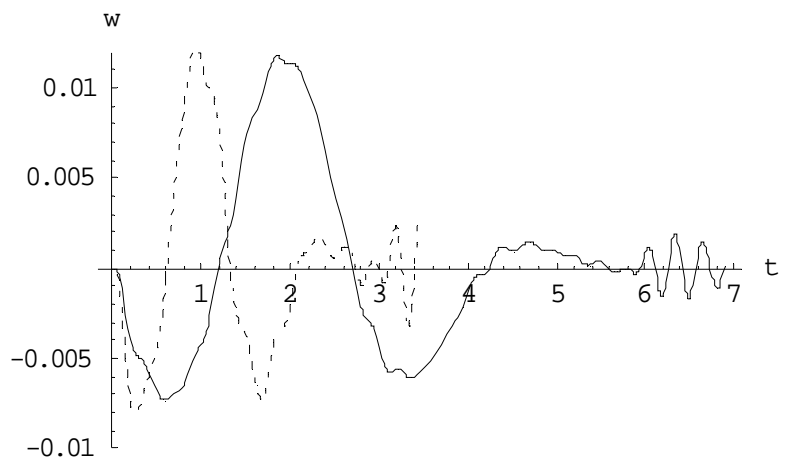

Fig. (8). Influence lines at $\mathrm{w}_{2}\left(\ell_{2} / 2, \mathrm{t}\right)$ for forced vibration of the beam.

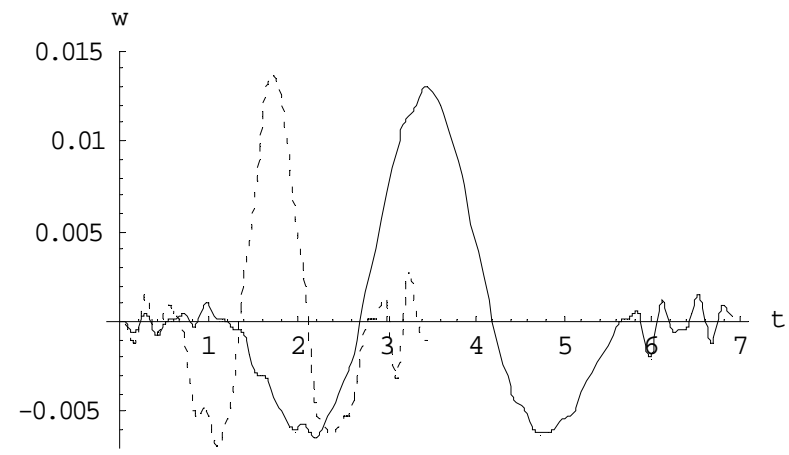

Fig. (9). Influence lines at $\mathrm{w}_{3}\left(\ell_{3} / 2, t\right)$ for forced vibration of the beam.

From Figs (7) through 9 one can see that the influence lines take maximum values with similar magnitude occurring 
though at different times depending on the moving force velocity.

\section{CONCLUSIONS}

A simple but very easy and efficient analytical method for obtaining reduced formulae in order to determine the eigenfrequencies and eigenmodes of multi-span continuous beams with spans of different lengths and bending rigidities is presented. The results can be readily employed for studying the dynamic response of multi-span beams subjected to any type of dynamic actions. From the examples studied, it is obvious that an optimum design of multi-span beams and subsequently a better overall dynamic behavior of continuous beams can be very easily achieved by employing the reduced formulae presented herein.

\section{REFERENCES}

[1] Frýba L. Vibrations of Solids and Structures under Moving Loads. Noordhoff Int. Publishing: Groningen 1972.

[2] Inglis CE. A Mathematical Treatise on Vibration in Railway Bridges. The University Press: Cambridge 1934.

[3] Hillerborg A. Dynamic Influences of Smoothly Running Loads on Simply Supported Girders. Kungl. Tkn. Högskolan, Stockholm 1951.

[4] Biggs JM, Suer HS, Louw JM. Vibration of simple-span highway bridges. Trans ASCE 1959; 124: 433-55.

[5] AASHO, Road Test, History and Description of Project. HRB Special Report 61A, NAS-NRC Publication 816, 1961.
[6] AASHO, Road Test, Bridge Research. HRB Special Report 61D, NAS-NRC Publication 953, 1962.

[7] Oehler LT. Vibration susceptibilities of various highway bridge type. ASCE J Struct Div 1957; 83: 845-64.

[8] Bily V. Analysis of System Consisting of Bridge Structure and Moving Vehicle by the Component Element Method. Proc. 2nd Int. Conf. on Traffic Effects on Structures and Environment, 377-84, 1991.

[9] Dmitriev AS. Dynamic of continuous multi-span beams under a moving force. Int Appl Mech 1982: 18(2): 179-86.

[10] Kong J, Cheung YK. Vibration of continuous beams using modified beam vibrating functions. Commun Num Methods Eng 1996: 12:107-14.

[11] Luo Y. Frequency analysis of infinite continuous beam under axial loads. J Sound Vibr 1998: 213(5): 791-800.

[12] Munirudrappa N, Iyengar DHN. Dynamic analysis of continuous span highway bridge. ISET J Earthquake Technol 1999: 36(1): 7384.

[13] Ichikawa M, Miyakawa Y, Matsuda A. Vibration analysis of the continuous beam subjected to a moving mass. J Sound Vibr 2000: 230(3): 493-506

[14] Greco A, Santini A. Dynamic response of a flexural non-classically damped continuous beam under moving loading. Comput Struct 2002: 80: 1945-53.

[15] Samaan M, Kennedy J, Sannah K. Dynamic analysis of curved continuous multiple-box girder bridges. J Bridge Eng 2007: 12(2): 184-93.

[16] Mehri B, Davar A, Rahmani O. Dynamic Green function solution of beams under a moving load with different boundary conditions. Sharif University of Technology, Trans B: Mech Eng 2009: 16(3): 273-9.

[17] Michaltsos GT, Sophianopoulos D, Kounadis AN. The effect of a moving mass and other parameters on the dynamic response of a simply supported beam. J Sound Vibr 1996: 191(3): 357-62.

Received: November 02, 2011

(C) Konstantakopoulos et al.; Licensee Bentham Open.

This is an open access article licensed under the terms of the Creative Commons Attribution Non-Commercial License (http://creativecommons.org/licenses/by-nc/3.0/) which permits unrestricted, non-commercial use, distribution and reproduction in any medium, provided the work is properly cited. 